\title{
Variation in cortical evoked responses as a function of performance criterion ${ }^{1}$
}

\author{
D. W. NIELSEN,2 D. C. TEAS, 3 AND R. P. IDZIKOWSKI \\ UNIVERSITY OF FLORIDA
}

Averaged evoked cortical responses (CER) from the scalp of human Ss were recorded within an experimental paradigm that permitted the performance criterion to be varied. The signals evoking the cortical responses were contingent upon $S$ 's pressing a button to bisect a temporal interval within certain tolerance limits. Under passive conditions averaged response waveforms lacked a second, late component that became prominent under temporal bisection conditions. The late component $P_{2}-N_{2}$ increased regularly in magnitude as the performance criterion was made more stringent. The effect of performance criterion on the earlier component, $N_{1}-P_{2}$, was neither as large nor as systematic as that shown by $\mathrm{P}_{2}-\mathrm{N}_{2}$.

Many investigators have shown that the waveform of the cortical evoked response (CER) recorded from the scalp of human $S$ s varies with the state of the $S$. The CER has been investigated in relation to psychological parameters, labeled variously: significance (Larson, 1960), vigilance (Ritter \& Vaughan, 1969), attention (Tecce \& Scheff, 1969; Donchin \& Cohen, 1967; Mast \& Watson, 1968; Satterfield, 1965; Garcia-Austt et al, 1964; Spong et al, 1965), decision (Debecker \& Desmedt, 1966; Davis, 1964), expectancy

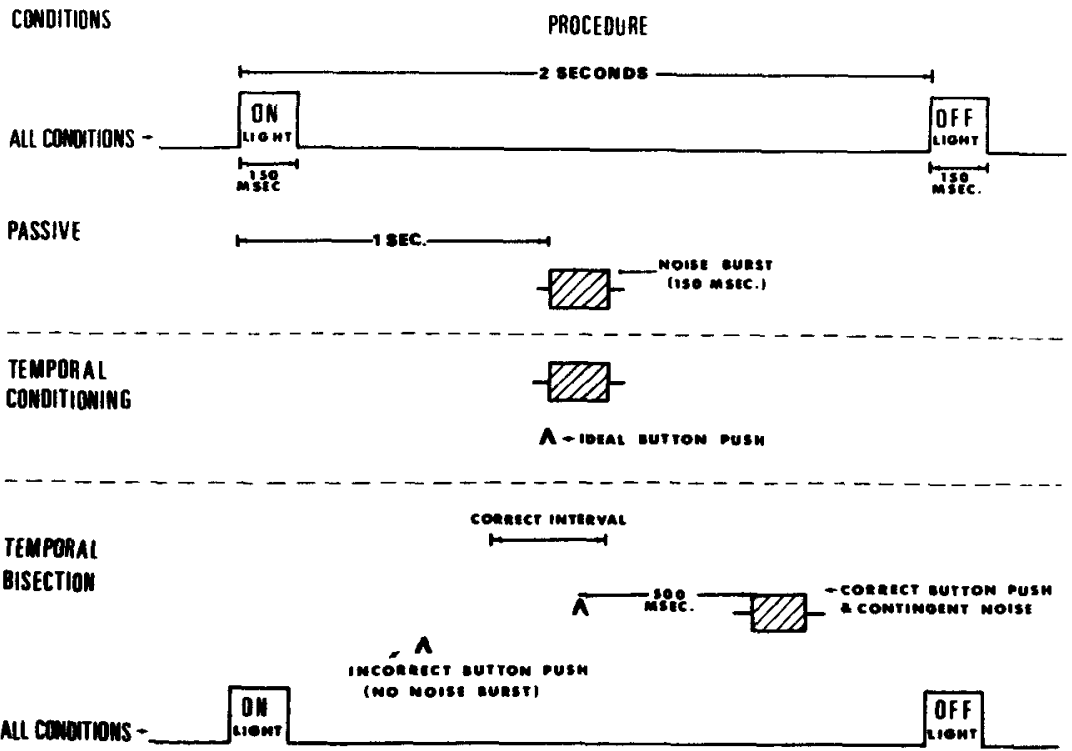
stimuli is significant. expanded or contracted systematically.

\section{PROCEDURE}

PROCEDURE
(Walter et al, 1964), contingency (Hillyard \& Galambos, 1967), and conditioning (Low et al, 1966). Although various properties of the CER waveform were emphasized and the investigators used different tasks in their experiments, there is general agreement that the relation of S's "psychological state" to the relevant

The objective of the study reported here was to assess variation in the CER, not in the sense of the labels enumerated above, but simply as a function of the levels of S's performance, where the delivery of the stimuli evoking the CER depended upon that performance. Ss were required to bisect a temporal interval with graded restrictions on the acceptability of their error. The signals were contingent upon S's temporal bisection occurring within specified tolerance limits that were

Throughout the experiment S's scalp activity was monitored con tinually with an oscilloscope and on-line averaging of responses. The data were also recorded on magnetic tape. Pulses representing the occurrence of the stimuli (light and noise burst), button pressed, incorrect responses, and voice markers were recorded on other tracks of the tape. The electrical activity$$
\text { the }
$$
the the same time as the onset of the noise the noise burst occurred at the exact

Fig. 1. Each $S$ received the experimental condition in order (top to bottom). Onand off-lights were present for all conditions. Passive: $\mathbf{S}$ received signals passively, no behavioral response occurred. Temporal conditioning: noise burst presented each trial, $S$ attempted to press button contiguously with onset of noise burst. Temporal bisection: noise burst contingent on correct bisection. This condition repeated three times with tolerance limits on acceptable error of S's bisection varied. If button press occurred within specified tolerances, a noise burst occurred $500 \mathrm{msec}$ following the correct button press. $A$ fourth condition was run, repeating the passive. 
midpoint of the 2-sec interval, that the temporal accuracy of his button press would be recorded for 50 trials, and that his accuracy should improve during the 50 trials. This phase of the experiment is treated, therefore, as a temporal conditioning (TC) phase.

The third phase of the experiment was the temporal bisection (TB) condition. In this condition, the 2-sec temporal interval was again marked off by the lights, but the noise burst was made contingent upon a correct temporal bisection of the interval between the on- and off-lights. $S$ was instructed to bisect the 2 -sec interval by pressing the button at the exact center of the interval. It was pointed out that the center of the interval occurred exactly at that point in time that had been marked by the onset of the noise burst in the two previous tasks. $S$ was informed that the accuracy of his temporal bisection would be recorded and that he would hear the noise burst $500 \mathrm{msec}$ after the button press if his accuracy was within certain temporal limits.

Each $S$ was run for three consecutive blocks of 50 trials in TB. Before each block of trials, he was given a short break and was informed of the amount of accuracy required to consider the response "correct" and thus for him to produce the noise burst. The three tolerance limits used were $\pm 75 \mathrm{msec}, \pm 106 \mathrm{msec}$, and $\pm 150 \mathrm{msec}$. Five Ss were run with increasing restriction on the acceptability of "correctness" and four Ss were run so that the tolerance limits became wider for each block of 50 trials. No differences in either performance or evoked responses were seen for the two groups.

The fourth phase of the experiment was another passive condition $\left(\mathbf{P}_{\mathrm{B}}\right)$ exactly like the first. Thus, for each $S$ in each condition scalp response activity was recoverable to three stimuli, the "on-light," the "off-light," and the noise burst. In the $\mathrm{P}_{\mathrm{A}}$ and $P_{B}$ conditions, $S$ 's role was that of a passive filter. In the $\mathrm{TC}$ condition, $S$ was an active element in the experiment, and in

Fig. 2. Averaged cortical evoked response waveforms for lights and noise burst under various experimental conditions. Passive $A$ and Passive $B$ show comparisons for the average of the first 6 trials and the last 6 trials for the 50-trial block. Acoustic noise bursts following correct temporal bisections produced CER waveforms that differ in magnitude and complexity as compared to CERs in passive conditions. The center column shows that the magnitude of CER to noise burst increases with stringency of criterion. $N$ for all responses is 6; sweep duration, 1 sec, all responses shown at same gain.
TB his performance determined whether or not the noise burst occurred. The three conditions therefore represent three different degrees of participation in the experiment by the $S$.

\section{Data Analysis}

Recovery of the average responses was carried out after the experimental session from the taped records. The off-line processing permitted the recovery of estimates of evoked activity, i.e., averages, to the noise burst and to the on- and off-lights. The noise burst was delayed from the button press by $500 \mathrm{msec}$ and bears no consistent temporal relation with the lights. For recovery of the averaged response to the noise burst, the computer was synchronized on S's button press. The trigger was then delayed by $400 \mathrm{msec}$ so that the sweep began $100 \mathrm{msec}$ before the noise burst.

For averaged responses to the on-light, the computer was triggered with a pulse representing the on-light. For responses to the off-light, the on-light trigger was delayed by $1,800 \mathrm{msec}$. All averages were computed for a 1-sec sweep duration (sample period).

For the TC section of the experiment, the latency of S's button press was recorded for each trial. From these latencies, trials were selected for which (1) the latencies closely approximated the onset of the noise burst, (2) the latencies < onset of the noise burst, and (3) the latencies $>$ onset of the noise burst. The distribution of latencies for each subgroup of responses from each $S$ fell within 10 msec. Our objective was to extract any deflections antedating the button press. No reliable indicators of such antedating activity were found. Perhaps the 10 -msec range of the distributions reduced synchrony so that any small, brief, but regular, deflections were obscured.

Although 50-trial blocks were run for the $\mathbf{P}_{\mathbf{A}}, \mathbf{P}_{\mathbf{B}}$, and $\mathrm{TC}$ phases of the experiment, average responses were based on subsets within the blocks. The objective was to sum as few samples as necessary to obtain an estimate of evoked activity. It was found that an $\mathrm{N}$ of six samples of evoked activity provided an optimum estimate. This made possible a comparison of evoked activity at the beginning of each block of 50 trials with that close to the end of each S0-trial block.

\section{RESULTS}

There are two types of signals in the experiment that evoke CERs: (1) the light pulses marking the beginning and end of each trial, and (2) the signal of principal interest, the acoustic noise burst. Both visual and acoustic signals were presented under all experimental conditions. The visual signals were never subject to control by S's performance. The acoustic signals in the contingent conditions were subject to such control and, in passive conditions, were not. The principal comparisons, then, are for the CERs to the acoustic signal in passive vs temporal bisection conditions, and within the temporal bisection condition for the three tolerance limits defining acceptable error. A secondary comparison is the CER to the regularly occurring visual signal, not under performance control, for passive and temporal bisection conditions. That is, is the effect of contingency (noise burst) general for all signals presented during the contingency condition or is the effect specific for the contingent signal?

Figure 2 shows average responses for one $S$ and contrasts CERs between passive and temporal bisection conditions for both the light pulses and the acoustic signal.

\section{Passive CERs}

The first row of Fig. $2\left(P_{A}\right)$ shows that the on-light and the noise burst produce CERs similar in magnitude at the beginning of the experimental session. The off-light, however, produces practically no discernible deflection in this example. For the on-light and noise burst, the CER for

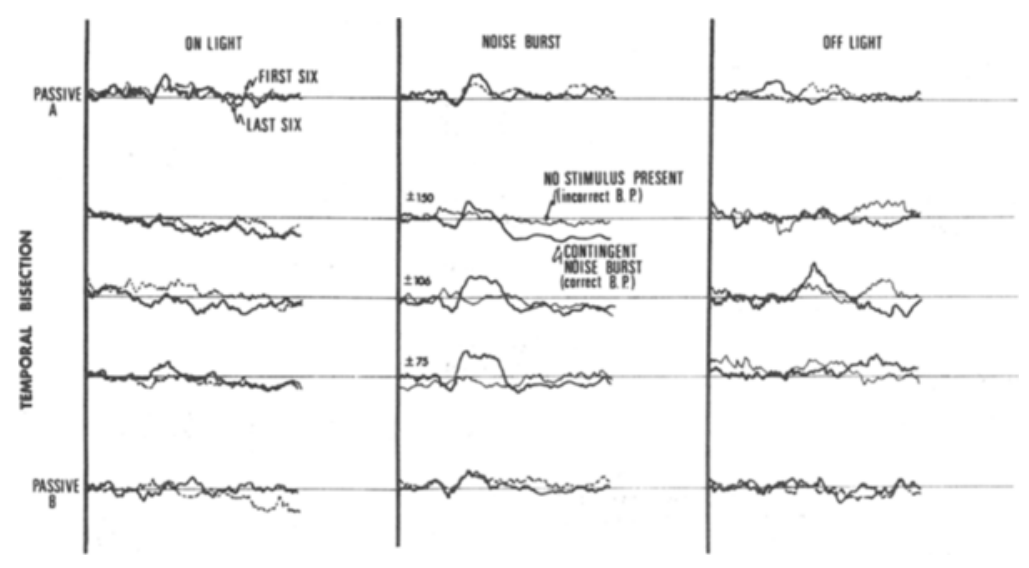


the first six trials is larger than that for the last six trials of the first 50-trial block (i.e., $\left.P_{A}\right)$. The waveform becomes smaller but shows little alteration in temporal features over the 50 trials. This habituation is further advanced particularly for the on-light in the final row of Fig. $2\left(\mathrm{P}_{\mathrm{B}}\right)$. The CER to the noise burst shows no further decrease in magnitude after the first 50-trial block.

\section{Contingent CERs}

The middle column of Fig. 2 (noise burst) shows that CER waveform is altered in the TB condition. Although the initial $\mathbf{N}_{1}-\mathbf{P}_{2}$ deflection changes only slightly, the later portions of the waveform increase in magnitude and are followed by a net negative deflection of the baseline. The positivity at $P_{2}$ is, in the averaged response, extended in time and, finally, drops to the negative value, i.e., below the baseline established prior to the onset of the response to the noise burst. The alteration in waveform occurs only when the noise burst is contingent upon S's performance and does not appear for either passive condition.

With respect to alteration in average response magnitude and waveform, the temporal conditioning phase falls between the passive and temporal bisection conditions. Figure 3 shows average (calculated) values for the two measurements based on the responses from nine Ss. The passive condition represents the responses from first passive conditions, chosen to avoid including the effects of habituation. Notice that the $P_{2}-N_{2}$ deflection is smaller than the $N_{1}-P_{2}$ deflection for the passive condition only.

The responses in the temporal conditioning phase show, on the average, a slight increment for both deflections, as contrasted with passive, but $P_{2}-N_{2}$ increased more than $\mathbf{N}_{1}-\mathbf{P}_{2}$. Within the temporal bisection condition, $\mathbf{P}_{\mathbf{2}}-\mathbf{N}_{\mathbf{2}}$ increased regularly in magnitude with the decrease in the tolerance limits that defined an acceptable temporal bisection by the $S$. Although $N_{1}-P_{2}$ also increased for the temporal bisection condition, the increment was less systematic than was the $\mathbf{P}_{2}-\mathbf{N}_{2}$ deflection.

Confidence limits were calculated for each deflection. Only the $\mathrm{P}_{2}-\mathrm{N}_{2}$ deflection for the \pm 75 -msec tolerance limit exceeded the $95 \%$ confidence interval. All other differences fell within the confidence interval to be expected on the basis of the variability in the magnitude of the averages.

Correct temporal bisections were well distributed throughout each block of 50 trials for the temporal bisection conditions. Since the noise bursts were contingent upon S's performance, the number of stimuli delivered decreased as the limit of tolerance defining correct button presses decreased. Figure 4 shows the number of correct button presses for each TB tolerance limit. As the tolerance limits increased, the number of correct bisections also increased. However, the magnitude of $\mathrm{N}_{2}-\mathrm{P}_{2}$ in the CER shows an inverse relation with the size of the tolerance interval for averages of six responses.

\section{DISCUSSION}

The different experimental conditions within the recording session produced the anticipated changes in the averaged evoked responses. In the initial passive condition, evoked response magnitudes to the noise burst decreased over trials, but when the signal was made contingent upon S's performance, evoked response magnitude increased and exceeded that elicited by the

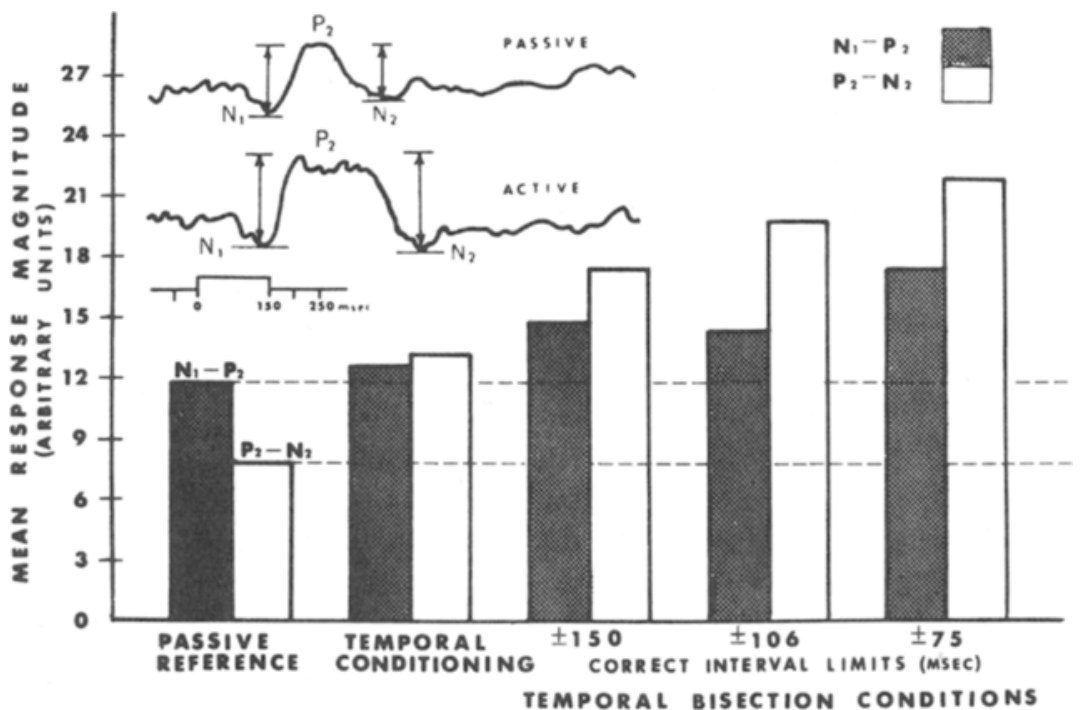

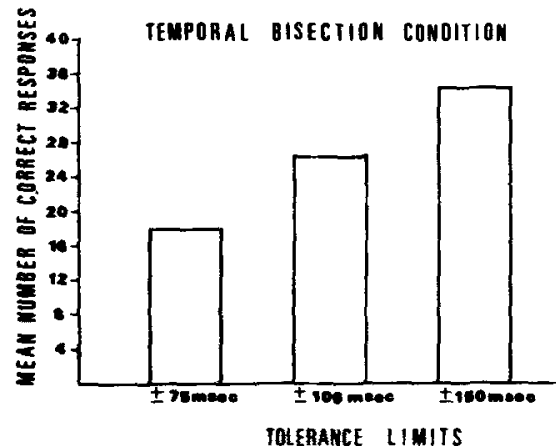

Fig. 4. Mean number of correct temporal bisections for nine Ss. Compare the increase in number of correct behavioral responses as tolerance limits widen with the decrease in $\mathbf{N}_{2}-\mathbf{P}_{2}$ magnitude shown in Fig. 3.

same signal during the passive condition. Not expected, however, was the additional strength of the $P_{2}-N_{2}$ component, which increased systematically with stringency of the criterion for acceptable performance.

The data demonstrate therefore that electrical activity evoked by an acoustic signal can be made to wax or wane by permitting or by withholding opportunity for S's performance to control the occurrence of the signal evoking the electrical response. Simply requiring $S$ to make a behavioral response (TC) prevents habituation for 50 trials-at least in this study-but did not produce as great an increment in response magnitude or alteration in response waveform as did temporal bisection.

During the TB condition, response magnitude to the acoustic signal increased but that to the on-light continued to decrease. That is, the enhancement of the averaged response to the contingent signal was contemporaneous with the decrement in the response to the invariant signal. Thus, enhancement is not a general arousal phenomenon, but is specific to the particular signal that is contingent upon the S's behavioral responses.

The inverse relation between magnitude of $\mathrm{P}_{2}-\mathrm{N}_{2}$ and number of correct responses is striking. The relation suggests that the temporal bisection procedure, utilizing a delayed sensory input as a sign of correct performance and to evoke the CER, will be a useful procedure for maintaining specified steady-state levels of performance over a prolonged period.

Fig. 3. Mean values of measurements indicated in inset for nine Ss. Note the regular increase in magnitude of $\mathbf{P}_{2}-\mathbf{N}_{2}$ component with temporal conditioning (self-imposed criterion) and increasing restriction on tolerance limit of $S$ error (E-imposed criteria). 


\section{REFERENCES}

DAVIS, H. Enhancement of evoked cortical potentials in humans related to a task requiring a decision. Science, 1964, 145, 182-183.

DEBECKER, J., \& DESMEDT, J. S. Rate of intramodality switching disclosed by sensory evoked potentials averaged during signal detection tests. Joumal of Physiology (London), 1966, 185, 52P-53P.

DOCHIN, E., \& COHEN, L. Averaged evoked potentials and intramodality selective attention. Electroencephalography \& Clinical Neurophysiology, 1967, 22, 537-546.

GARCIA-AUSTT, E., BOGACZ, J., \& VANZULLI, A. Effects of attention upon visual e voked response. Electroencephalography \& Clinical Neurophysiology, 1964, 17, 136-143.

HILLYARD, S. A., \& GALAMBOS, R. Effects of stimulus and response contingencies on a surface negative slow potential shift in man. Electroencephalography \& Clinical Neurophysiology, 1967, 22, 297-304.

IRWIN, D. A., KNOTT, J. R., MCADAM, D. W., \& REBERT, C. S. Motivational determinants of the contingent negative variation. Electroencephalography \& Clinical Neurophysiology, 1966, 21, 538-543.
LARSON, L. Correlation between the psychological significance of stimuli and the magnitude of the startle blink and evoked EEG potentials in man. Acta Physiologica Scandinavica, $1960,48,-76-294$.

LOW, M. D., BORDA, R. R., FROST, J. D., Jr., \& KELLAWAY, P. Surface-negative slow potential shift associated with conditioning in man. Neurology, 1966, 16, 771-782.

MAST, T. E., \& WATSON, C. S. Attention and auditory evoked responses to low detectability signals. Perception \& Psychophysics, 1968, 4, 237-240.

McADAM, D. W., IRWIN, D. A., REBERT, C. S., \& KNOTT, J. R. Conative control of the contingent negative variation. Electroencephalography \& Clinical Neurophysiology, 1966, 21, 194-195.

RITTER, W., \& VAUGHAN, H. G., JR. Average evoked responses in vigilance and discrimination: A reassessment. Science, 1969 164, 326-328.

SATTERFIELD, J. H. Evoked cortical responses enhancement and attention in man. A study of response to auditory and shock stimuli. Electroencephalography \& Clinical Neurophysiology, $1965,19,470-475$

SPONG, P., HAIDER, M., \& LINDSLEY, D. B.
Selective attentiveness and cortical evoked responses to visual and auditory stimuli. Science, $1965,148,395-397$.

TECCE, J. J., \& SCHEFF, N. M. Attention reduction and suppressed direct-current potentials in the human brain. Science, 1969, 164, 331-333.

WALTER, W. G., COOPER, R., ALDRIDGE, V. J., MCCALLUM, W. C., \& WINTER, A. L. Contingent negative variation: An electrical sign of sensorimotor association and expectancy in the human brain. Nature, 1964 , $203,380-384$.

\section{NOTES}

1. The research reported here was supported by Grants NB-05475, NB-07287, NB-06459. Thanks are due also to $K$. Decker and $K$. Lam for their assitance in data processing.

2. Address: Communications Science Laboratory, University of Florida, Gainesville, Florida 32601 .

3. Also Department of Psychology, Department of Physiology.

(Accepted for publication October 20, 1969.) 